\title{
EFFET PATHOGÈne DES EXOTOXINES DE 11 VARIÉTÉS APPARTENANT AU GROUPE DE BACILLUS THURINGIENSIS BERLINER, SUR L'ABEILLE DOMESTIQUE
}

\author{
Pathogene Wirkung der Exotoxine von elf zur Gruppe Bacillus Thurin- \\ giensis BERLINER gehörenden Varietäten auf die Honigbiene (Apis \\ mellifica $L$.)
}

Oldrich HARAGSIM et Jirina VANKOVA*

Institut de Recherches sur l'Abeille,

Dol, p. Libcice n. Vlt., Tchécoslovaquie

* Institut Entomologique de l'Académie tchécoslovaque des Sciences, Prague.

\section{SUMMARY}

\section{PATHOGEN EFFECT OF EXOTOXINS OF ELEVEN MICROBE VARIETIES, BELONGING}

TO THE (C BAGILLUS THURINGIENSIS ) BERLINER GROUP, ON HONEY-BEES

The paper presents the results of the study concerning the effect of the exotoxins of some microbe varieties of the Bacillus thuringiensis Berl. group on the imagoes of the honey-bee. The exotoxins were isolated and purified in the laboratories of the Czechoslovak Academy of Sciences. The method employed for this purpose was developed by Sebesta, Horská and Vankova $(1966,1969)$. The total number of exotoxins tested was 11.

Exotoxins, applied as injections to the thorax, affected the summer bee generation in the following order : cazaubon-the average duration of bee life after application- 1,7 day, thuringiensis $-1,9$ day, anduze 2,0 days, gelechiae- $-2,1$ day, alesti $-2,3$ day, bombycis $-2,4$ day, entomocidus $-2,6$ day, sotto $-2,9$ day, plebeja 3,4 day, dendrolimus $-3,8$ day and galleriae $-4,0$ days.

Like in the preceding trials with the spore crystalline material, the autumn bee generation was found more resistant to exotoxin.

After the peroral application of technical exotoxin in the concentration $1: 1$ (50\% sugar sirup : techn. toxin) the average duration of bee life was 5,1 day. When the concentration was $1: 3$, the duration of life was 6,5 day, concentration $1: 6-7,3$ day, concentration $1: 9$ $-9,4$ day, and $1: 18-8,7$ day. On the average, the bees to which technical exotoxin was applied lived 3,7 day.

Wasps of the species Paravespula fed technical exotoxin in sugar sirup were killed at the concentration of $1: 3$ after 9,7 day, at $1: 6$ after 9,9 day, at $1: 9$ after 10,6 day and at 1 : 18 after 9,3 day, on an average. Wasps were found more resistant to the effects of exotoxins.

Our trials did not demonstrate the hypothesis that the microbe Bacillus thuringiensis might be one of the causative agents of bee paralysis during honey-flow. 


\section{RÉSUME}

Les auteurs ont étudié l'effet des exotoxines de certaines variétés de Bacillus thuringiensis Berl. sur les imagos de l'abeille domestique. Les exotoxines étaient isolées et purifiées dans les laboratoires de l'Académie tchécoslovaque des Sciences en appliquant la méthode élaborée par Sebesta, Horska et Vankova $(1966,1969)$. Au total on a testé 11 exotoxines.

Sur la génération des abeilles d'été les exotoxines appliquées par injection dans le thorax, exerçaient leur effet dans l'ordre décroissant suivant : cazaubon - la durée de vie moyenne des abeilles à la suite de l'application des exotoxines était de 1,7 jour, thuringiensis - de 1,9 jour, anduze - de 2,0 jours, gelechiae - de 2,1 jours, alesti - de 2,3 jours, bombycis - de 2,4 jours, entomocidus - de 2,6 jours, sotto - de 2,9 jours, plebeja - de 3,4 jours, dendrolimus - de 3,8 jours et galleriae - de 4,0 jours.

La génération des abeilles d'automne se montre plus résistante à l'exotoxine tout comme dans les essais antérieurs effectués en utilisant le matériel constitué de spores et de cristaux.

En effectuant par voie buccale l'application de l'exotoxine technique à la concentration $1 / 1$ (50\% de sirop : $50 \%$ d'exotoxine technique), la longueur moyenne de la vie des abeilles était de 5,1 jours; à la concentration $1 / 3:$ de 6,5 jours, à la concentration $1 / 6:$ de 7,3 jours, à la concentration $1 / 9: 9,4$ jours et à la concentration $1 / 18:$ de 8,7 jours.

Les abeilles auxquelles on a injecté l'exotoxine technique, survivaient à la suite de l'application en moyenne pendant 3,7 jours.

Les guêpes du genre Paravespula, nourries d'exotoxine technique incorporée dans le sirop, ont péri à la concentration $1 / 3$, en moyenne après 9,7 jours, à la concentration $1 / 6:$ en 9,9 jours, à la concentration $1 / 9:$ en 10,6 jours et à la concentration $1 / 18:$ en 9,3 jours. Les guêpes sont plus résistantes aux effets des exotoxines.

L'hypothèse selon laquelle Bacillus thuringiensis pourrait être un des agents du “ mal noir " des abeilles, n’a pas été confirmée au cours de nos essais.

\section{INTRODUCTION}

Les variétés de bactéries, appartenant au groupe de Bacillus thuringiensis Berl., produisent plusieurs substances qui sont toxiques pour beaucoup d'espèces d'insectes. Dans un travail antérieur (HARAgsim et VANKova, 1968) nous avons étudié comparativement les effets des endotoxines (cristaux) et des spores pathogènes de 12 variétés de Bacillus thuringiensis sur l'abeille domestique et son couvain : il a été montré que les différentes variétés présentent des degrés différents de toxicité pour l'abeille domestique. Comme facteur important de la pathogénie nous avons considéré l'exotoxine non spécifique dont l'élaboration a lieu au cours de la croissance bactérienne sous forme soluble dans le milieu de culture. Nous avons admis qu'au cours du processus de production il peut demeurer dans la préparation sèche finale (spores + cristaux d'endotoxine) une certaine quantité d'exotoxine, dont la présence abrège la durée de vie des abeilles soumises aux expériences. Nous n'avons pu cependant répondre à la question de savoir quel effet exerce l'exotoxine sur les abeilles car, à cette époque-là, on n'avait pas encore isolé chez nous la substance en question.

En 1968 les chercheurs de l'Académie des Sciences tchécoslovaque ont réussi à mettre au point une technique permettant l'isolement des exotoxines de Bacillus thuringiensis. Nous avons alors obtenu une certaine quantité d'exo- 
toxine pure et simultanément l'exotoxine comprise dans l'élément surnageant du milieu de culture (exotoxine technique), ce qui nous a permis de vérifier leur effet sur l'abeille domestique.

Bacillus thuringiensis Berl. a longtemps été considéré comme une substance non nocive pour les abeilles. GuKasjan (1958) a cependant montré que la bactérie peut provoquer une mortalité brusque chez l'abeille si la culture est ajoutée à leur nourriture à une concentration assez forte. KRIEG et HERFs (1963) poursuivant les essais de GUKASJAN sur une plus grande échelle étudièrent la toxicité des différentes fractions obtenues au cours de la culture du microbe. Ils montrèrent que ce sont surtout les fractions qui comprennent l'exotoxine secrétée par le germe dans le milieu nutritif qui sont toxiques pour l'abeille. L'exotoxine ne s'est manifestée comme élément toxique que lorsqu'elle était distribuée aux abeilles dans la nourriture régulièrement pendant 5 à 10 jours. Martouret et Euverte (1964) et Cantwell, Knox, Lehnert et Michael (1964) sont arrivés aux mêmes conclusions.

Au cours d'essais effectués en cagettes, Cantwell, Knox, Lehnert et MICHAEL ont montré que l'exotoxine de Bacillus thuringiensis variété thuringiensis détruit $50 \%$ des abeilles en expérience et cela déjà après sept jours, chaque abeille ayant absorbé en moyenne $0,63 \mathrm{mg}$ d'exotoxine. Ils ont été les premiers à attirer l'attention sur les effets toxiques de l'exotoxine pour le couvain d'abeille. A la suite de l'application de l'exotoxine à la concentration de $10 \mathrm{mg}$ par $\mathrm{ml}$, les jeunes larves ont toutes péri au bout de cinq jours. L'action de l'exotoxine sur les larves plus âgées était beaucoup plus faible, car après son application $30 \%$ du couvain a survécu dans les rayons, $11 \%$ produisant des imagos normaux. Les préparations mélangées d'endotoxine, de spores et d'exotoxine ont eu pour effet, aussi bien au cours des essais en cagettes que dans les essais semi-pratiques, de provoquer la mortalité très rapide des abeilles. En cagettes les abeilles ont péri dans un délai de sept jours; pendant les essais semi-pratiques il se produisit après quinze jours un affaiblissement très sensible des colonies, soumises à la préparation enrichie par la fraction d'exotoxine, tandis qu'une dose identique de préparation technique (endotoxine + spores) ne provoquait aucune manifestation comparable d'affaiblissement.

Dans notre étude précédente (Haragsim et Vankova, 1968) nous avons comparé l'effet pathogène des endotoxines et des spores de douze variétés appartenant au groupe Bacillus thuringiensis sur les imagos et le couvain de l'abeille domestique. Nous avons démontré que c'est l'exotoxine non spécifique, produite par les variétés en quantités différentes, qui peut être le facteur important de la pathogénie des variétés. Dès lors nous avons signalé qu'en testant les préparations du commerce il faut prendre surtout en considération le procédé de production, la variété utilisée et la quantité d'exotoxine qui demeure, au cours de la production, dans la préparation sèche. 
Les chercheurs de l'Académie des Sciences thécoslovaque, K. Sebesta, K. Horska et J. Vankova ont réussi en 1966 à mettre au point un procédé permettant d'isoler et de purifier l'exotoxine thermostable de Bacillus thuringiensis, variété gelechiae. En appliquant le même procédé on a obtenu ensuite les exotoxines des autres souches de Bacillus thuringiensis et celles-ci constituèrent le matériel de base de nos essais effectués sur les abeilles.

\section{L'objectif du présent travail consistait à déterminer la toxicité des exo-} toxines des différentes souches de Bacillus thuringiensis et à comparer leur effet pathogène sur les imagos de l'abeille domestique. Nous avons étudié simultanément l'action de ces exotoxines sur un autre insecte vivant en société, à savoir la guêpe commune Paravespula vulgaris $\mathbf{L}$.

\section{MATÉRIEL ET MÉTHODE}

Les différentes souches de Bacillus thuringiensis Berl., étaient cultivées dans un milieu liquide au citrate (Cantwell G.-E., Heimpel A.-M., Thompson M.-J., 1964) à la température de $29^{\circ} \mathrm{C}$, en utilisant un agitateur à 105 oscillations par minute. Les fermenteurs, d'une capacité de $500 \mathrm{ml}$, étaient remplis de $60 \mathrm{ml}$ de milieu de culture et inoculés, toujours deux à deux, avec une culture de vingt-quatre heures sur agar oblique. Après 48 heures de culture dans l'agitateur, on centrifugeait la biomasse. Les exotoxines étaient absorbées sur charbon et éluées à l'alcool après lavage. Les produits de concentration étaient soumis à l'électrophorèse sur papier. Ensuite, on éluait les matières qui se déplaçaient en bandes de façon analogue aux exotoxines dans la direction de l'anode et s'absorbaient dans la zone de l'exotoxine ou dans son voisinage. En procédant de la sorte, à partir de $50 \mathrm{ml}$ de liquide de culture de chacune des souches, on a obtenu $3 \mathrm{ml}$ d'exotoxine concentrée (voir liste des souches testées au tableau 1).

Les abeilles mises en expérience provenaient du rucher de l'Institut de recherches apicoles de Dol et appartenaient à la race carniolienne (Apis mellifera carnica Pollm.). Elles étaient conservées à l'étuve dans les mêmes conditions qu'au cours des essais antérieurs (HaRAGsim et VANKOVA, 1968).

Les toxines des différentes souches de Bacillus thuringiensis Berl. étaient administrées aux abeilles adultes par injection ou par voie buccale. Avant l'injection on a anesthésié sur de la glace sèche, les abeilles âgées de 4 à 6 jours. On a injecté dans les muscles thoraciques de chaque abeille $0,005 \mathrm{ml}$ soit d'exotoxine stérile, soit de toxine technique comprise dans la partie surnageante du milieu de culture. Les abeilles témoins ont reçu une injection d'un même volume d'eau, apyrogène, stérile. On a ensuite placé les abeilles dans les cagettes, les laissant pendant 10 à 20 minutes à l'air frais, pour permettre le réveil. Plus tard, elles ont été transportées dans l'étuve et nourries de sirop à $50 \%$ et de pelotes fraîches de pollen en rayon. Les quelques abeilles dont le réveil après l'injection tardait trop ou qui présentaient des symptômes de paralysie étaient éliminées.

Pour l'application par voie buccale des exotoxines, on ménageait aux abeilles les mêmes conditions et on contrôlait quotidiennement la mortalité. On alimentait les nourrisseurs de nourriture fraîche, à savoir de sirop additionné d'exotoxine à des concentrations $1 / 1,1 / 3$, $1 / 6,1 / 9$, et $1 / 18$, ou bien d'exotoxine technique initiale non concentrée, contenant environ $20 \mathrm{gg}$ de matière active par $\mathrm{ml}$.

Au cours des essais on a utilisé deux groupes d'abeilles, à savoir des abeilles jeunes, dont l'éclosion avait eu lieu au mois de juin (abeilles dites d'été) et des abeilles jeunes dont l'éclosion avait eu lieu au mois d'août (abeilles dites d'automne) que l'on considère en général comme des abeilles à longue durée de vie et plus résistantes que les abeilles d'été. Par voie buccale on n'utilisait que des abeilles d'automne. 
TaBL. 1. - Variêtés testées de Bacillus thuringiensis Berl.

TAB. 1. - Die untersuchten Stämme von Bacillus thuringiensis Berl.

\begin{tabular}{|c|c|c|}
\hline $\begin{array}{l}\text { Variété } \\
\text { Stamm }\end{array}$ & $\begin{array}{c}\text { Hóte } \\
\text { Wirt }\end{array}$ & $\begin{array}{c}\text { Refférence } \\
\text { Bezug }\end{array}$ \\
\hline 1. galleriae $\ldots \ldots \ldots \ldots \ldots \ldots \ldots \ldots \ldots$ & Galleria mellonella & $159 / 1$ \\
\hline 2. plebeja $\ldots \ldots \ldots \ldots \ldots \ldots \ldots \ldots \ldots$ & Cicada plebeia & $204 / 1$ \\
\hline 3. dendrolimus $\ldots \ldots$. & Dendrolimus sibiricus & $306 / 2$ \\
\hline 4. bombycis $\ldots \ldots \ldots \ldots \ldots \ldots \ldots \ldots$ & Bombyx mori & $393 / \mathrm{T}$ \\
\hline 5. entomocidus $\ldots \ldots \ldots \ldots \ldots \ldots \ldots$ & Aphomia gularis & $459 / 2$ \\
\hline 6. sotto $\ldots \ldots \ldots \ldots \ldots \ldots \ldots \ldots \ldots$ & Bombyx mori & $461 / 1$ \\
\hline 7. alesti $\ldots \ldots \ldots \ldots \ldots \ldots \ldots \ldots$ & Bombyx mori & $463 / 1 \mathrm{~T}$ \\
\hline 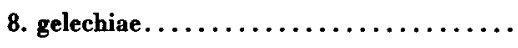 & Gelechia gossypiella & $\mathbf{5 5 5}$ \\
\hline 9. cazaubon ..... & Pyrausta nubilalis & $\mathbf{5 5 7}$ \\
\hline 10. anduze $\ldots$. & Bombyx mori & $558 / Z T$ \\
\hline 11. thuringiensis $\ldots \ldots \ldots \ldots \ldots \ldots \ldots \ldots$ & Plodia interpunctella & $601 / T$ \\
\hline
\end{tabular}

\section{RESULTATS}

Il ressort des résultats obtenus, résumés de façon synoptique aux tableaux $2,3,4$, et 5 que les exotoxines des souches examinées appartenant au groupe de Bacillus thuringiensis Berl., sont toxiques pour l'abeille à des degrés différents. Les abeilles d'expérience de la génération estivale (jeunes abeilles de juin) sont mortes pour la plupart dès les quatre premiers jours à la suite de l'injection des exotoxines dans les muscles thoraciques, à la dose de $0,005 \mathrm{ml}$. Seules quelques abeilles ont vécu plus de quatre jours, et une seule survivait encore le neuvième jour.

Les abeilles issues de la génération d'automne manifestent une plus grande résistance aux toxines de $B$. thuringiensis, comme l'ont montré déjà les premiers essais, où l'on utilisait le matériel cristaux-spores. Elles sont également plus résistantes aux exotoxines. En effet, après l'injection des exotoxines elles vivaient en moyenne plus longtemps que les abeilles de la génération d'été, certaines d'entre elles demeurant encore en vie le $12^{\mathrm{e}}$ jour après l'application. Chez les abeilles d'automne la mortalité élevée n'était jamais atteinte dès les premiers jours de l'essai, comme c'était le cas pour la génération d'été.

Les exotoxines des différentes souches de Bacillus thuringiensis exercent leurs effets sur les abeilles d'une façon assez différente. Chez les abeilles d'été l'exotoxine de la souche cazaubon montre l'effet le plus toxique; après son 
o. haRAGSIM, Jirina VANKova

\begin{tabular}{|c|c|c|c|c|c|c|c|}
\hline & 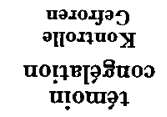 & in & ถึ & & - & en & 1 \\
\hline & 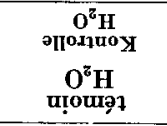 & ติ & 字 & & $\boldsymbol{N}$ & $r$ & - \\
\hline & 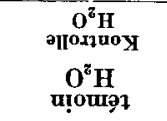 & หน & ถิ & & N & 1 & 1 \\
\hline & -q6ฐ & ถึ & $\stackrel{\infty}{\text { \& }}$ & & $\circ$ & สี & $\stackrel{\sim}{\sim}$ \\
\hline & 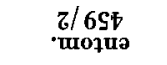 & ติ & 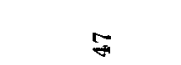 & & $r$ & 2 & 0 \\
\hline & $\underset{\text { oznpus }}{\mathbf{y} / \text { /gss }}$ & है & \%) & & $a$ & ల్లి & $\varrho$ \\
\hline & $\begin{array}{l}\mathrm{I} / 6 S \mathrm{SI} \\
-\mathrm{I}\end{array}$ & ถิ & 5 & & 0 & er & $v$ \\
\hline & $\begin{array}{c}\text { SSS } \\
\text { 'पุอə[ə, }\end{array}$ & ถึ & ติ & & $\infty$ & ஜำ & $\stackrel{\Xi}{\beth}$ \\
\hline 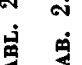 & 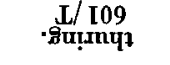 & 禺 & 18 & & $\Rightarrow$ & $\stackrel{\infty}{\text { ஸे }}$ & 0 \\
\hline & - LSS & 모 & $\underset{+}{+\infty}$ & & లి & $\infty$ & + \\
\hline & 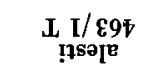 & 8 & \& & & $\stackrel{2}{2}$ & $e^{\prime}$ & $\Xi$ \\
\hline & $\begin{array}{l}\text { I/ I97 } \\
\text { o7fos }\end{array}$ & ถิ & घิ & & $\Xi$ & 0 & $\approx$ \\
\hline & $\begin{array}{l}\mathrm{t} / \mp 0 Z \\
\cdot \mathbf{q}]^{d}\end{array}$ & หิ & 9 & & $\infty$ & in & $a$ \\
\hline & $\begin{array}{l}\text { z/90\& } \\
\text { трuәр }\end{array}$ & ํㅗ & हे & & 0 & m & er \\
\hline & 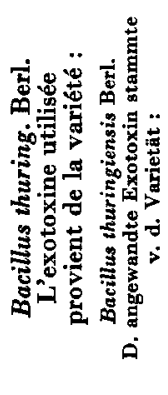 & 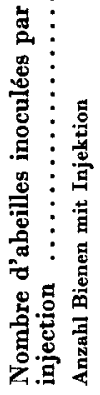 & 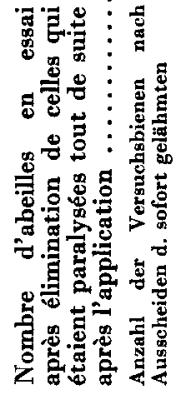 & 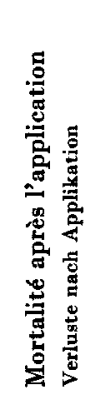 & 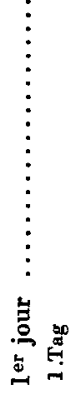 & 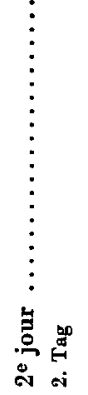 & 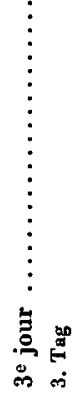 \\
\hline
\end{tabular}




\begin{tabular}{|c|c|c|c|c|c|c|c|}
\hline 1 & 1 & I & + & lo & 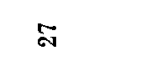 & 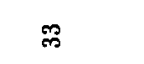 & \\
\hline 1 & 1 & 1 & $\pi$ & $q$ & $\underset{\Im}{\infty}$ & 只 & 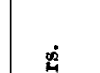 \\
\hline 1 & 1 & 1 & N & $\$$ & $\stackrel{\infty}{\infty}$ & $\stackrel{m}{\circ}$ & 4 \\
\hline$\infty$ & 1 & 1 & $\$$ & 1 & ov & + & $\bar{z}$ \\
\hline 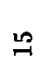 & 1 & 1 & F & I & oi & + & 㐫 \\
\hline 1 & 1 & 1 & \& & 1 & oे & $\infty$ & \\
\hline ลे & N & 1 & f & 1 & $\stackrel{\circ}{+}$ & $\infty$ & ty \\
\hline 1 & 1 & 1 & in & I & $\overrightarrow{\text { s }}$ & $\infty$ & \\
\hline 1 & 1 & 1 & $\$$ & I & $\overrightarrow{7}$ & $\infty$ & \\
\hline 0 & 1 & 1 & $\stackrel{\infty}{q}$ & 1 & $\tilde{i}$ & $\sigma$ & \\
\hline$\cong$ & 1 & 1 & q & 1 & ${ }_{i j}$ & + & \\
\hline$\cong$ & $\infty$ & 1 & 용 & I & $\vec{i}$ & $\infty$ & \\
\hline $\mathscr{ల}$ & 1 & 1 & s & I & of & + & 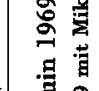 \\
\hline r & $a$ & 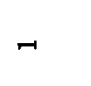 & $\therefore$ & 1 & $\underset{\infty}{\infty}$ & $a$ & ه్ \\
\hline$\frac{5}{\circ}$ & $\begin{array}{c}\vdots \\
\vdots \\
\vdots \\
\vdots \\
\end{array}$ & $\begin{array}{c}\vdots \\
\vdots \\
\vdots \\
\vdots \\
\vdots \\
\vdots \\
\vdots \\
\vdots \\
\vdots \\
\vdots \\
\vdots \\
\vdots\end{array}$ & 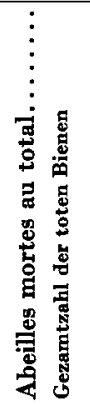 & 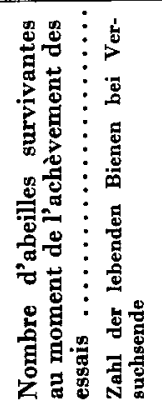 & 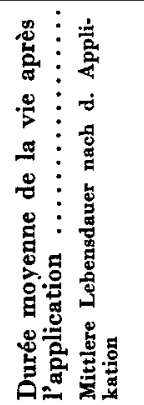 & 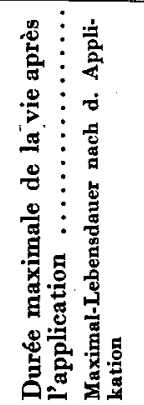 & 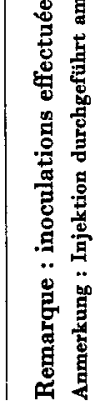 \\
\hline
\end{tabular}


ค่

\begin{tabular}{|c|c|c|c|c|c|c|c|}
\hline 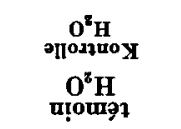 & 요 & จิ & & 1 & 1 & 1 & 1 \\
\hline 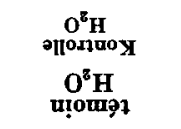 & ק్ & 오 & & N & I & 1 & 1 \\
\hline $\begin{array}{l}\stackrel{L}{L} / \varepsilon 6 \varepsilon \\
\cdot q u 0 q\end{array}$ & 户్ & 요 & & - & $N$ & $\infty$ & 1 \\
\hline 6/6St & 우 & $\bar{n}$ & & 1 & + & 1 & N \\
\hline$\underset{\text { əznpus }}{\mathrm{J} / 88 \mathrm{~S}}$ & 요 & 요 & & $\infty$ & 1 & $\mathbf{N}$ & $\infty$ \\
\hline${ }_{\cdot{ }^{\prime}}^{\mathrm{I} / 6 \mathrm{SI}}$ & ్ㅏ & 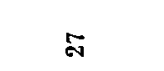 & & $\nabla$ & 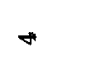 & $\infty$ & on \\
\hline$\underset{\text { प甲 }}{\text { SSg }}$ & ణ్ & సิ & & is & 2 & in & 1 \\
\hline 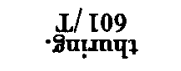 & ద్లి & $\stackrel{\infty}{\sim}$ & & $\infty$ & $\stackrel{\infty}{\sim}$ & $\mathbf{N}$ & 1 \\
\hline qqnezes & 요 & สิ & & $\Rightarrow$ & $\infty$ & in & - \\
\hline 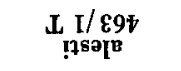 & 요 & ק్ల & & in & $\infty$ & 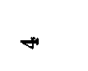 & 1 \\
\hline I/ 197 & 88 & 요 & & 1 & 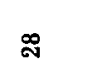 & $r$ & $r$ \\
\hline $\mathbf{L}_{\cdot \mathbf{q}}^{\mathbf{I} / \boldsymbol{t} \mathbf{f} \mathbf{d}}$ & 요 & శิ & & 1 & $-r$ & 1 & 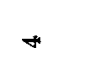 \\
\hline $\begin{array}{l}\text { 7/90ॄ } \\
. \text { Ipuəp }\end{array}$ & ర్లి & बิ & & r & 1 & 1 & $a$ \\
\hline 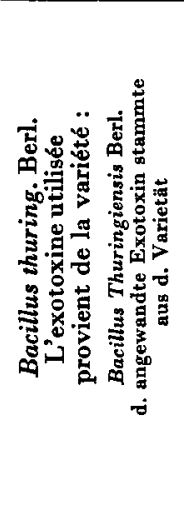 & 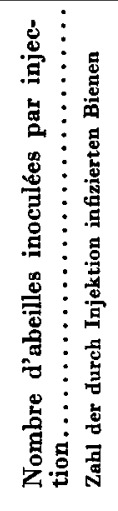 & 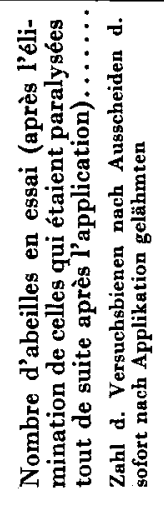 & 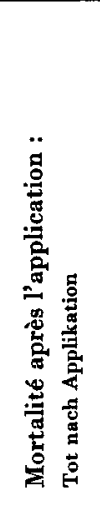 & 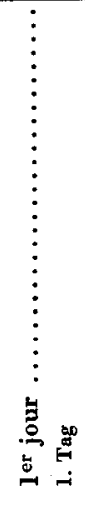 & $\begin{array}{c}\vdots \\
\vdots \\
\vdots \\
\vdots \\
\vdots \\
\vdots \\
\vdots \\
\vdots \\
\text { 岂 } \\
\text { ஸึ } \\
\text { ஸे }\end{array}$ & 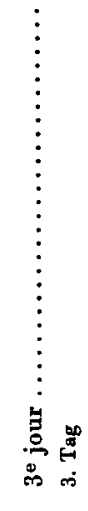 & 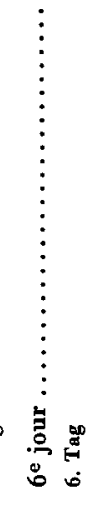 \\
\hline
\end{tabular}




\begin{tabular}{|c|c|c|c|c|c|c|c|c|c|c|}
\hline | & - & 1 & 1 & 1 & $N$ & $\infty$ & : & 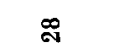 & 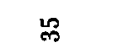 & \\
\hline-1 & 1 & m & 1 & 1 & 1 & $r$ & ศึ & กัง & లొ & \\
\hline 웅 & N & + & 1 & 1 & 0 & 요 & 1 & $\stackrel{0}{\circ}$ & $\stackrel{\mathcal{I}}{ }$ & \\
\hline N & 0 & 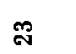 & 1 & 1 & 1 & $\tilde{m}$ & I & $\stackrel{\infty}{=}$ & $a$ & \\
\hline$\infty$ & 0 & $N$ & - & 1 & $\infty$ & 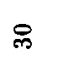 & 1 & 3 & 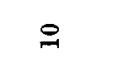 & $\stackrel{\circ}{\stackrel{\circ}{0}}$ \\
\hline n & is & - & $N$ & 1 & 1 & $\tilde{\sim}$ & 1 & : & $\mathcal{\exists}$ & 壱 \\
\hline I & 1 & 1 & 1 & 1 & 1 & సิ & 1 & ने & $\infty$ & 品跑 \\
\hline I & 1 & I & I & 1 & 1 & $\stackrel{\infty}{\sim}$ & 1 & $\stackrel{\infty}{-}$ & $\infty$ & \\
\hline 1 & - & 1 & N & 1 & -1 & ลิ & I & $\hat{\mathrm{N}}$ & $\cong$ & 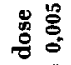 \\
\hline 1 & - & 1 & in & エ & 1 & हे & I & $\Rightarrow$ & $\Rightarrow$ & 焉要 \\
\hline- & is & 1 & 1 & 1 & 1 & in & 1 & oi & $\infty$ & 遌 \\
\hline$a$ & 0 & $\infty$ & 0 & 1 & 1 & ลิ & 1 & $\tilde{\sigma}$ & 용 & 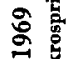 \\
\hline o & N & $m$ & $\infty$ & $\boldsymbol{N}$ & | & ને & 1 & $\stackrel{\pi}{\therefore}$ & $=$ & 要 \\
\hline $\begin{array}{l}\vdots \\
\vdots \\
\vdots \\
\vdots \\
\vdots \\
\vdots \\
\vdots \\
\vdots \\
\vdots \\
\vdots \\
\vdots \\
0 \\
0\end{array}$ & $\begin{array}{l}\vdots \\
\vdots \\
\vdots \\
\vdots \\
\vdots \\
\vdots \\
\vdots \\
\vdots \\
\vdots \\
\vdots \\
\vdots \\
\vdots \\
D_{\infty}^{\circ}\end{array}$ & $\begin{array}{l}\vdots \\
\vdots \\
\vdots \\
\vdots \\
\vdots \\
\vdots \\
\vdots \\
\vdots \\
\vdots \\
\vdots \\
. \\
\vdots \\
\vdots\end{array}$ & 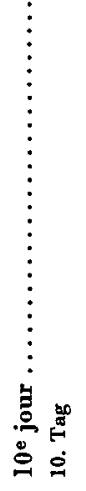 & 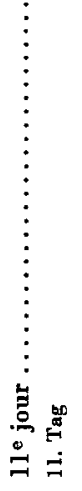 & 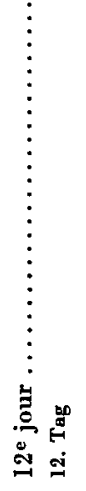 & 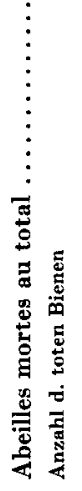 & 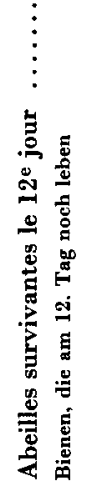 & 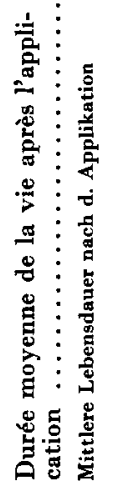 & 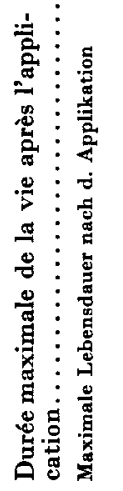 & 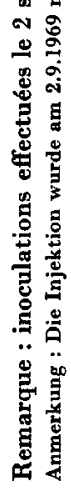 \\
\hline
\end{tabular}


application les abeilles ont péri en moyenne au bout de 1,7 jours. Ensuite venaient la souche thuringiensis : 1,9 jour, et la souche anduze : 2,0 jours. La longévité moyenne la plus grande s'est trouvée chez les abeilles ayant reçu une injection de l'exotoxine de la souche galleriae : 4,0 jours, de la souche dendrolimus : 3,8 jours et de la souche plebeja $: 3,4$ jours.

Sur la génération des abeilles d'automne l'exotoxine de la souche thuringiensis exerçait l'effet le plus toxique, la longévité moyenne après son application étant de 1,8 jour; venaient ensuite la souche sotto : 2,6 jours, la souche cazaubon : 2,9 jours et la souche gelechiae : 2,9 jours.

Vu la consommation importante des aliments au cours des essais effectués en cagettes sur les abeilles, on ne disposait pas d'assez d'exotoxine pure concentrée, et, pour cette raison on distribuait par voie buccale l'exotoxine technique du liquide fermenté qui contenait environ $20 \mu \mathrm{g} / \mathrm{ml}$ de matière toxique active. Après les essais effectués per os et pour pouvoir comparer correctement l'effet de l'exotoxine technique, on a appliqué l'exotoxine technique par injection à quatre séries d'abeilles (172 individus). Leur longévité moyenne à la suite de cette application était quelque peu plus élevée que celle obtenue en appliquant la matière concentrée. Elle s'élevait à 3,7 jours, égalant presque, quant à son effet, celui de la toxine de la souche dendrolimus.

Les abeilles auxquelles on a appliqué per os l'exotoxine technique dans le sirop à $50 \%$, à la concentration $1 / 1$, vivaient après l'application en moyenne 5,1 jours; à la concentration $1 / 3$ jours : 6,5 jours à la concentration $1 / 6: 7,3$ jours; à la concentration $1 / 9: 9,4$ jours et à la concentration $1 / 18: 8,7$ jours. La consommation moyenne de la nourriture par abeille était plus élevée lorsqu'on distribuait un sirop plus concentré.

Les abeilles mortes par suite des effets toxiques des exotoxines des variétés de Bacillus thuringiensis ne présentaient pas de symptômes extérieurs spécifiques d'infection. Le noircissement de l'armature chitineuse était mat et peu marqué. Les abeilles ne perdaient pas leurs poils sur la partie dorsale des segments de l'abdomen et ne présentaient pas d'effilochage des bords membraneux des ailes. Les abeilles épuisées s'attroupaient au fond des cagettes, tremblaient, aspiraient avidement l'eau ou le sirop dilué, et se montraient très apathiques. Dans l'hémolymphe, nous n'avons observé la présence d'aucun microbe et par conséquent il ne s'agissait pas de septicémie.

L'hypothèse selon laquelle Bacillus thuringiensis pourrait être un des agents de la maladie des abeilles dites “ mal noir " n'a pas été confirmée au cours des essais. Les exotoxines des différentes souches de Bacillus thuringiensis sont cependant toxiques pour les abeilles.

Comparativement aux abeilles, les guêpes se montrent plus résistantes à l'effet de l'exotoxine technique administrée par voie buccale. A la suite de 
l'introduction de l'exotoxine dans la nourriture, les guêpes vivaient en moyenne de 9,3 à 10,6 jours, soit comparativement deux fois plus longtemps que les abeilles. De plus il faut noter que la consommation moyenne de nourriture par les guêpes était beaucoup plus élevée que celle des abeilles. L'exotoxine injectée dans le thorax des guêpes agissait au contraire d'une façon très intense de sorte qu'après la narcose toutes les guêpes sont restées totalement paralysées, succombant dans les deux premiers jours.

\section{DISCUSSION}

Du point de vue chimique, l'exotoxine est définie comme analogue d'un nucléotide comprenant l'adénine, le ribose, le glucose, l'acide allarique et le groupe phosphatique (Sebesta, Horska, Vankova, 1969). En étudiant le mécanisme de l'effet de l'exotoxine sur des animaux d'expérience, on a montré à la différence des affirmations antérieures, que c'est une substance toxique

TABL. 4. - Effet de l'exotoxine technique sur les abeilles - application par voie buccale.

TAB. 4. - Wirkung der technischen Exotoxine auf die Bienen. Orale Anwendung.

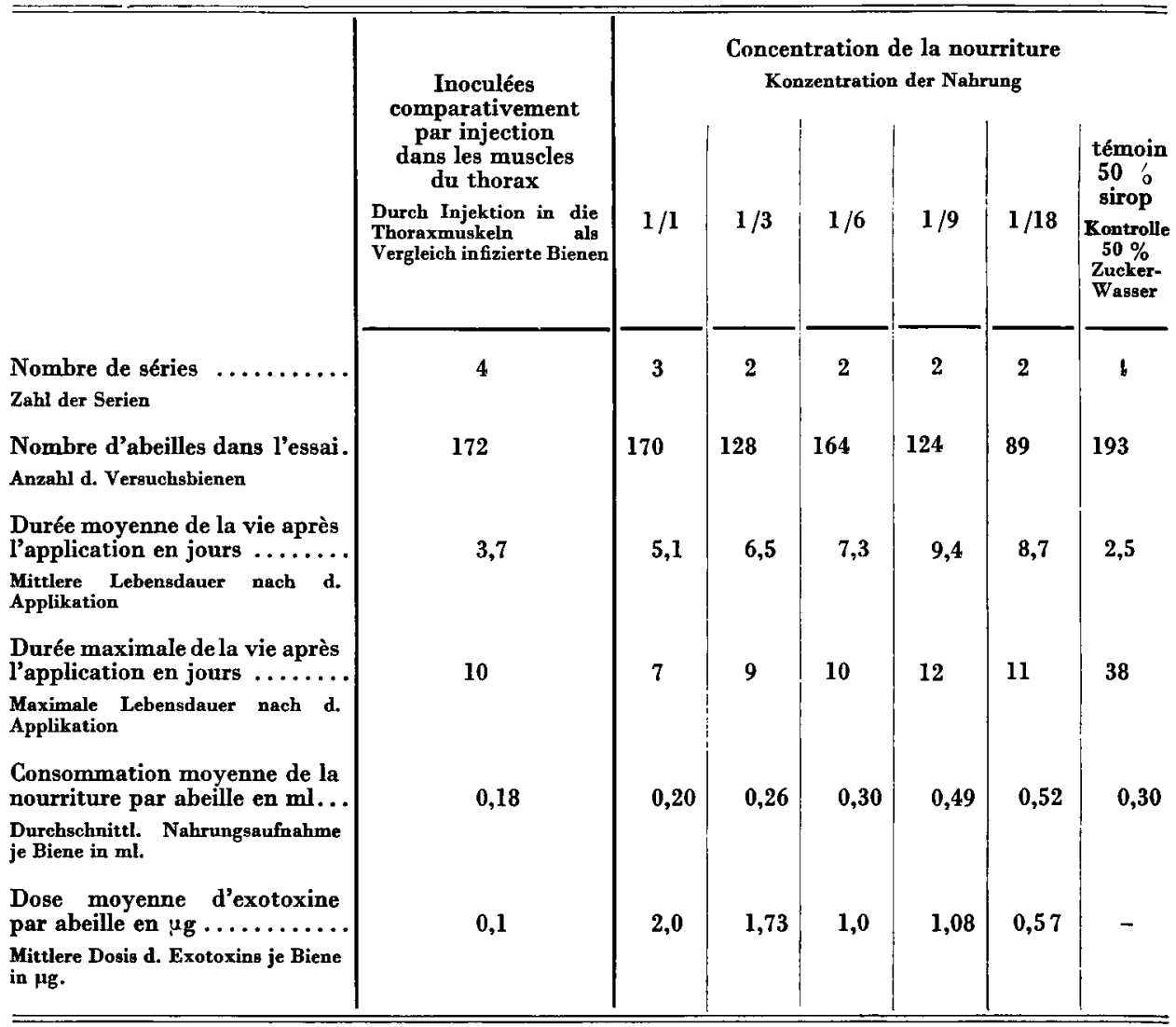


non seulement pour les insectes, mais aussi pour les mammifères (souris).

Au cours des essais sur les abeilles nous avons constaté que l'exotoxine appliquée aux abeilles par injection dans les muscles thoraciques à la dose de $0,1 \mu \mathrm{g}$ est beaucoup plus efficace que $2,0 \mu \mathrm{g}$ d'exotoxine administrée aux abeilles per os. Les abeilles auxquelles on a administré l'exotoxine technique par injection dans les muscles vivaient en moyenne 3,7 jours et les abeilles qui ont absorbé l'exotoxine technique dans la nourriture vivaient en moyenne 5,1 jours, bien que la dose moyenne d'exotoxine par abeille fût considérablement plus forte. Dans les conditions d'absorption de l'exotoxine par voie buccale il se produit apparemment une déphosphorylation enzymatique et de ce fait aussi une réduction de la toxicité de l'exotoxine.

Au cours des essais effectués sur les guêpes de l'espèce Paravespula vulgaris, les différences étaient encore plus nettes.

Nous avons réalisé à titre d'orientation, la déphosphorylation de l'exotoxine in vitro, au moyen des phosphomono-estérases obtenues à partir du tube

TaBL. 5. - Effet de l'exotoxine technique sur les guêpes (Paravespula vulgaris L.). Application effectuée par voie buccale.

TAB. 5. - Wirkung der technischen Exotoxine auf Wespen (Paravespula vulgaris L.). Orale Anwendung.

\begin{tabular}{|c|c|c|c|c|c|}
\hline & \multicolumn{5}{|c|}{$\begin{array}{c}\text { Concentration de la nourriture } \\
\text { Konzentration der Nahrung }\end{array}$} \\
\hline & $1 / 3$ & $1 / 6$ & $1 / 9$ & $1 / 18$ & $\begin{array}{c}\text { Témoin } 50 \% \text { sirop } \\
\text { Kontrolle } 50 \% \\
\text { Zucker-Wasser }\end{array}$ \\
\hline $\begin{array}{l}\text { Nombre de séries d'expériences } \ldots \ldots \ldots \\
\text { Zahl d. Versuchsserien }\end{array}$ & 2 & 2 & 2 & 2 & 2 \\
\hline $\begin{array}{l}\text { Nombre de guêpes dans l'essai } \ldots \ldots \ldots \\
\text { Zahl. d. Versuchstiere }\end{array}$ & 99 & 84 & 77 & 97 & 86 \\
\hline $\begin{array}{l}\text { Durée moyenne de la vie après appli- } \\
\text { cation en jours } \ldots \ldots \ldots \ldots \ldots \ldots \ldots \ldots \ldots \\
\text { Mittlere Lebensdauer nach Applikation }\end{array}$ & 17 & 9,9 & 10,6 & 9,3 & 32 \\
\hline $\begin{array}{l}\text { Durée maximale de la vie après appli- } \\
\text { cation en jours } \ldots \ldots \ldots \ldots \ldots \ldots \ldots \ldots \ldots \ldots \ldots \ldots \\
\text { Maximale Lebensdauer nach Applikation }\end{array}$ & 9,7 & 19 & 20 & 20 & 48 \\
\hline $\begin{array}{l}\text { Consommation moyenne de la nourri- } \\
\text { ture par guêpe en ml } \ldots \ldots \ldots \ldots \ldots \ldots\end{array}$ & 0,65 & 0,28 & 0,57 & 0,70 & 0,52 \\
\hline $\begin{array}{l}\text { Durchschnittl. Nahrungsaufnahme je Wespe } \\
\text { in ml. }\end{array}$ & & & & & \\
\hline $\begin{array}{l}\text { Dose moyenne de toxine comprise dans } \\
\text { la nourriture en } \mathrm{ug} \ldots \ldots \ldots \ldots \ldots \ldots \ldots \\
\text { Durchschnittl. Giftmenge in d. Nahrung in } \mu \mathrm{g}\end{array}$ & 4,3 & 3,0 & 1,26 & 0,77 & - \\
\hline
\end{tabular}

Nota : le $48^{\mathrm{e}}$ jour après le début de l'essai, le témoin comptait 27 abeilles $(31 \%)$

Anmerkung : Am 48. Tag nach Versuchsbeginn lebten noch 27 Kontrolltiere $=31 \%$. 
digestif des abeilles et des guêpes. A la suite de la dégradation obtenue avec la phosphatase alcaline, la toxicité de l'exotoxine déphosphorylée a été réduite chez les abeilles à $40 \%$ et chez les guêpes à $20 \%$ de la valeur initiale. En présence de la phosphatase acide. par contre, l'exotoxine n'a subi aucune déphosphorylation ni chez les abeilles ni chez les guêpes et conservait sa toxicité initiale.

L'aptitude relativement faible de l'exotoxine à la dégradation par les phosphomono-estérases qui prédominent dans le tube digestif des abeilles et des guêpes justifie comment l'exotoxine conserve ses propriétés en passant par le tube digestif et exerce son action toxique lorsqu'elle est administrée par la voie buccale.

Les guêpes ont absorbé au cours des essais une quantité plus grande de nourriture, et par conséquent aussi un volume plus grand d'exotoxine que les abeilles, mais vivaient en moyenne plus longtemps qu'elles. Nous estimons que l'efficacité des exotoxines dans le tube digestif des guêpes a été réduite sous l'action de la phosphotase alcaline. En administrant l'exotoxine par injection (comme il ne s'agit que d'une seule série nous ne l'indiquons pas aux tableaux comme résultat), on a vu périr toutes les guêpes au bout de 36 heures au plus tard. Ce qui est caractéristique dans ce cas c'est que les guêpes sont restées étendues sur le fond de la cagette, comme paralysées, ne manifestant qu'une activité minime.

Dans notre travail antérieur (Haragsim, Vankova, 1968) nous nous sommes occupés de la toxicité du matériel sporulé de Bacillus thuringiensis pour l'abeille et son couvain et dans le présent travail nous considérons celle des exotoxines. Dans les deux travaux nous avons accordé notre attention au rapport éventuel entre Bacillus thuringiensis et le « mal noir » des abeilles que beaucoup considèrent comme une septicémie. Cette manifestation survient souvent à la suite de la piqûre de parasites (Toumanoff, 1959 - Hostounsky, 1963 et autres); elle se développe dans les larves de beaucoup d'espèces d'insectes et même à la suite de piqûres dues aux acariens (par exemple du genre Pyemotes) (Weiser, 1966). D'ailleurs ce type d'infection ne se développe pas toujours dans l'insecte après une blessure, mais souvent aussi à partir de l'intestin. WeISER (1966) fait ressortir qu'en cas d'infection de l'insecte par Bacillus thuringiensis, la septicémie est induite par la microflore intestinale banale dans l'intervalle entre l'intoxication de l'hôte par la toxine et le développement final du microbe dans le corps de l'hôte.

Au cours de nos essais et de nos observations nous n'avons pas cependant remarqué de mortalité des abeilles due à la septicémie ni la manifestation des symptômes spécifiques du « mal noir ». Dans les frottis de l'hémolymphe on n'a jamais détecté de microbes. La véritable cause de la mort rapide des abeilles et des guêpes en relation avec Bacillus thuringiensis, doit être recherchée dans 
l'intoxication provoquée par l'exotoxine. S'il arrive qu'on trouve une septicémie provoquée par ce microbe, il ne s'agit alors que d'une infection associée atypique.

De même, il faut remarquer qu'au cours des essais nous avons utilisé l'exotoxine à une concentration élevée. Les préparations produites à l'échelle industrielle à base de Bacillus thuringiensis ne peuvent contenir que des résidus d'exotoxines. Pendant la production, il importe de veiller cependant sur la pureté de telles préparations.

Reçu pour publication en novembre 1972.

Eingegangen im November 1972.

\section{ZUSAMMENFASSUNG}

1. Die Verfasser haben ihre Untersuchungen über die pathogene Wirksamkeit der Exotoxine gewisser Stämme von Bacillus thuringiensis Berliner in bezug auf die Imagines der Honigbiene (Apis mellifica L.) fortgesetzt. Die Exotoxine wurden in der Tschechoslowakischen Akademie der Wissenschaften nach der Methode von Sebesta, Horska und Vankova (1966 1969) isoliert und rein dargestellt.

Von elf Stämmen wurden die Exotoxine isoliert und getestet.

2. Die in die Thorax-Muskulatur injizierten Exotoxine der verschiedenen Stämme wirkten auf die Sommerbienen ihrer Giftigkeit nach in abnehmender Folge :

- cazaubon, mittlere Lebensdauer nach Behandlung mit dem Exotoxin : 1,7 Tage; thuringiensis : 1,9 Tage; anduze : 2,0 Tage; gelechiae : 2,1 Tage; alesti : 2,3 Tage; bombycis : 2,4 Tage; entomocidus : 2,6 Tage; sotto 2,9 Tage; plebeja : 3,4 Tage; dendrolimus : 3,8 Tage; galleriae : 4 Tage.

3. Die Herbstbienen erwiesen sich als widerstandsfähiger gegen die Exotoxine in der gleichen Weise, wie es sich im Verlauf früherer Versuche mit sporuliertem Material gezeigt hatte. Das Exotoxin wirkt nach seiner Giftigkeit in abnehmender Folge :

- thuringiensis, mittlere Lebensdauer der Bienen nach Applikation des Exotoxins : 1,8 Tage; sotto : 2,6 Tage; cazaubon: 2,9 Tage; gelechiae : 2,9 Tage; galleriae : 6,0 Tage; anduze : 6,3 Tage; alesti : 7,1 Tage; dendrolimus : 7,4. Tage; bombycis : 7,6 Tage; entomocidus : 7,8 Tage; plebeja : 9,2 Tage.

4. Bei oraler Anwendung technischer Exotoxine in einer Konzentration von $1: 1(50 \%$ Zuckerwasser, $50 \%$ technische Exotoxine) beträgt die mittlere Lebensdauer der Bienen nach der Applikation 5,1 Tage; in einer Konzentration von $1: 3=6,5$ Tage; von $1: 9=9,4$ Tage; von $1: 18=8,7$ Tage.

Die Bienen, denen technische Exotoxine injiziert wurden, lebten im Durchschnitt 3,7 Tage nach der Injektion.

5. Wespen, denen Exotoxine in Zucherwasser verabreicht wurden, starben bei einer Konzentration von $1: 3$ nach 9,7 Tagen; von $1: 6$ nach 9,9 Tagen; von $1: 9$ nach 10,6 Tagen und von 1 : 18 nach 9,3 Tagen. Die Wespen sind demnach den Exotoxinen gegenüber widerstandsfähiger als die Bienen.

6. Die Hypothese, nach der Bacillus thuringiensis einer der Krankheitserreger der sogen. Schwarzsucht der Biene sein könnte, wurde durch diese Untersuchungen nicht bestätigt. 


\section{RÉFERENCES BIBLIOGRAPHIQUES}

BAILEY L., 1961. Infectious diseases of Honey-bee. London $176 \mathrm{p}$.

Bretschko J., 1969. Das Problem der bakteriellen Septikämie der Honigbiene (Apis mellifera L.). Bienenwelt $11: 264-267$.

Burnside C.-E., 1945. The cause of paralysis of honeybees. Amer, Bee J. (85) : 354-355, 363.

Cantwell G.-E., Heimpel A.-M., Thompson M.-J., 1964. The production of an exotoxin by various crystal-forming bacteria, related to Bacillus thuringiensis var. thuring. Berl., J. Insect. Pathol. (6) : 466-480.

Cantwell G.-E., Knox D.-A., Michael A.-S., 1964. Mortality of Honey-bees (Apis mellifera L.) fed exotoxin of Bac. thuringiensis Berl., J. Insect. Pathol. (6) : 532-536.

Dreher K., 1940. Zur Frage der Schwarzsucht der Honigbiene. Deutsch. Imkerführer 14, (6) : 93.

GUKASJAN A.B., 1958. Vosprimcivost pcel $\mathbf{k}$ vozbuditelju bolezni sibirskogo selkoprjada. - Ptchelövodstvo 35 (11): 46-48.

Haragsim O., 1966. Medovice a vcely. - Praha, 186 p.

Haragsim O., Vankova J., 1968. Effet pathogène comparé de 12 souches de Bacillus thuringiensis Berl. sur l'abeille domestique et son couvain. Ann. Abeille 11 (1) : 31-40.

Haragsim O., Vankova J., 1971. L'action pathologique des endo et exotoxines de certaines souches du microbe Bacillus thuringiensis Berl. sur l'abeille mellifère et son couvain. Congr. internat. Apicult. 32. 1971. Moscou, rés. rapports, 49-50.

Harahsim O., Vankova J., 1968. Biologicka ochrana vceliho dila. Vcelarstvi (3) : 36-37.

Коцомгјес N.-G., 1960. Rolj much sarkofagii $v$ razprostraneniji septicemii sibirskogo selkoprjada. Izv. Akad. Nauk SSSR (2) : 189-211.

KrIEg A., 1967. Neues über Bacillus thuringiensis und seine Anwendung. Mitt. Biol. Bundesanst. f. Land - und Forstwirtsch., Berlin-Dahlem. 125 : 1-106.

Krieg A., Herfs W., 1963. Uber Wirkung von Bacillus thuringiensis auf Bienen. Entomol. exp. appl. (6) : $1-9$.

Martouret D., Euverte G., 1964. The effect of Bacillus thuringiensis Berl. preparations on the Honey Bee under conditions of forced feeding. J. Insect Pathol. (6) : 198-203.

Poltev V-I., 1949. Etologia letnej gibeli pcel na lesnych pasekach Primorskogo kraja. Bolezni pcel, Trudy XXVII VASCHNIL.

Poltev V.-I., 1969. Mikro flora nasekomych. Novosibirsk, $270 \mathrm{p}$.

Rothenbuhler W., Kulincevic J., Stairs G., 1969. An adult honeybee disease usually unrecognized. Glean. Bee Cult. (6) : 329-331.

Svoboda J., Haragsimova L., Hanko J., Haragsim O., 1968. Nemoci a skudei vcely medonosné. Praha, $210 \mathrm{p}$.

Sebesta K., Horska K., Vankova J., 1969. Inhibition of de novo RNA synthesis by the insecticidal exotoxin of Bac. thuring. var. gelechiae. Coll. Czech. Chem. Comm. (34) : 1786-1791.

Sebesta K., Horska K., Vankova J., 1969. Isolation and properties of the insecticidal exotoxin of Bac. hturing. var. gelechiae. Coll. Czech. Chem. Comm. (34) : 891-900.

Toumanoff C., 1959. Observation concernant le rôle probable d'un prédateur dans la transmission d'un bacille aux chenilles. Ann. Inst. Pasteur (96) : 108-110.

Weiser J., Nemoci hmyzu. Praha, 1966, 554 p. 